\title{
Síndrome opsoclonus-mioclonus en paciente con cáncer de ovario
}

\author{
E. RUBIO NAZÁBAL, J. MAREY LÓPEZ, P. ÁLVAREZ PÉREZ', S. LÓPEZ FACAL, \\ L. ALONSO MAGDALENA
}

Servicio de Neurología. Hospital Juan Canalejo. ${ }^{~}$ Medicina Familiar y Comunitaria. Consultorio La Torre. La Coruña

\author{
OPSOCLONUS-MYOCLONUS SYNDROME IN PATIENT WITH OVA- \\ RIAN CANCER
}

\begin{abstract}
RESUMEN
El síndrome opsoclonus-mioclonus (SOM) es un trastorno poco frecuente que ha sido descrito como manifestación paraneoplásica, fundamentalmente asociado al neuroblastoma en niños y a tumores de mama y pulmón en adultos. La asociación con cáncer de ovario es excepcional habiéndose descrito sólo dos casos en la literatura; en ambos el patrón clínico fue similar precediendo el SOM a la neoplasia y mejorando con el tratamiento de la misma. A diferencia de estos en el caso que presentamos, el SOM se manifestó posteriormente al diagnóstico de cáncer de ovario, después del tratamiento de quimioterapia y radioterapia, mejorando con corticoides y clonazepan.
\end{abstract}

PALABRAS CLAVE: Cáncer de ovario. Opsoclonus-mioclonus. Síndrome paraneoplásico.

\begin{abstract}
The opsoclonus-myoclonus syndrome is a rare entity as a paraneoplastic disorder usually associated to neuroblastoma in children and breast cancer or oat-cell lung carcinoma in adults. The association of opsoclonus-myoclonus syndrome and ovarian carcinoma is very unusual, to our knowledge there is only two cases reported in the literature. In both of them the opsoclonus-myoclonus syndrome preceded the neoplasy, improving with its treatment. In our patient opsoclonus began after ovarian cancer diagnosis, after chemotherapy and radiotherapy, improving with corticoid and clonazepan therapy.
\end{abstract}

KEY WORDS: Ovarian carcinoma. Opsoclonus-myoclonus. Paraneoplastic syndrome.

Rubio Nazábal E, Marey López J, Álvarez Pérez P, López Facal S, Alonso Magdalena L. Síndrome opsoclonus-mioclonus en paciente con cáncer de ovario. An Med Interna (Madrid) 2003; 20: 370-372.

\section{INTRODUCCIÓN}

El opsoclonus es un raro y distintivo desorden de la motilidad ocular caracterizado por la presencia casi continua de movimientos sacádicos conjugados, involuntarios, arrítmicos, multidireccionales y de gran amplitud en todas las direcciones de la mirada. Este trastorno se presenta a menudo asociado a inestabilidad para la marcha con ataxia truncal y mioclonias afectando a las cuatro extremidades, tronco y cabeza constituyendo el síndrome opsoclonus-mioclonus (SOM). El SOM es un síndrome poco frecuente que ha sido descrito como manifestación paraneoplásica, fundamentalmente asociado a neuroblastoma en niños y a cáncer de mama y pulmón en adultos (1).

Presentamos el caso de una paciente que presentó el SOM como manifestación paraneoplásica de cáncer de ovario.

\section{CASO APORTADO}

Se trata de una paciente de 56 años que seis años antes había sido diagnosticada de Cistoadenocarcinoma papilar seroso de ovario estadio III, siendo tratada con cirugía (histerectomía más doble anexectomía) y quimioterapia (seis ciclos de cisplatino: $600 \mathrm{mg} / \mathrm{m}$ y ciclofosfamida: $600 \mathrm{mg} / \mathrm{m}$ ). Desde entonces, la paciente era seguida regularmente en consultas externas sin evidencia de recidiva tumoral. Cuatro meses antes del ingreso la enferma es diagnosticada de metástasis cerebral única parietal izquierda profunda no susceptible de tratamiento quirúrgico; se realiza biopsia que es concluyente con metástasis de carcinoma epitelial y es tratada con quimioterapia (cisplatino y paclitaxel) y posteriormente con radioterapia.

La paciente ingresa, tres meses después del tratamiento de quimioterapia y tras haber finalizado el tratamiento de radioterapia, por cuadro de siete días de evolución de sacudidas irregulares, de gran amplitud en extremidades superiores, en ocasiones lanzando objetos de las manos, con afectación posterior de miembros infe-

Trabajo aceptado: 10 de noviembre de 2002 
riores e intensa inestabilidad para la marcha que la obligaba a permanecer en cama. No relataba deterioro del nivel de conciencia ni cuadro viral previo. En la exploración la paciente estaba afebril, consciente, desorientada en espacio y tiempo, irritable, sin signos meníngeos, presentaba movimientos oculares continuos con sacudidas irregulares de gran amplitud en todas direcciones con predominio en el plano horizontal que la producían incapacidad para fijar la vista y que persistían con los ojos cerrados. Asimismo, se apreciaba temblor cefálico continuo. El resto de pares craneales eran normales así como el tono, fuerza, sensibilidad y ROT; el reflejo cutáneo plantar era flexor bilateral; la paciente presentaba sacudidas irregulares de gran amplitud en las cuatro extremidades que aumentaban con los movimientos voluntarios, interfiriéndolos. Además, se observaba ataxia truncal y de extremidades que la impedía la marcha.

Los estudios realizados fueron: hemograma: eritrocitos: 3,64 x 10(9)/l, Hemoglobina: 11,5 g/dl, Hematocrito: 36,2\%, VCM: 99,6 fl, leucocitos y plaquetas normales; Bioquímica: LDH: 480 U/l y GGT: 77 U/l, siendo el resto (glucemia, electrolitos, urea, creatinina, bilirrubina e inmunoglobulinas) normal; líquido cefalorraquídeo: no tenía células, proteínas $132 \mathrm{mg} / \mathrm{dl}$, glucosa 84 $\mathrm{mg} / \mathrm{dl}$ e inmunoglobulinas normales, no presentaba células malignas. La investigación de sustancias tóxicas en sangre y orina fue negativa. Los cultivos y serologías descartaron infección vírica, bacteriana o fúngica. El opsoclonus fue confirmado por electronistagmografía. El EEG mostró un enlentecimiento generalizado de la actividad de fondo sin actividad epileptiforme. La radiografía de tórax y la TAC toraco-abdominal no presentaban alteraciones. La RMN cerebral demostraba reducción de la lesión parietal (Fig. 1). Se realizó estudio de anticuerpos antineuronales anti-Yo, anti Ri y anti-Hu en suero y LCR que no fueron detectados.

La fue tratada con clonazepam $(0,5 \mathrm{mg} / 8 \mathrm{~h})$ y dexametasona (dosis inicial de16 mg/24 h). La evolución fue satisfactoria con mejoría progresiva, habiendo disminuido ostensiblemente los movimientos involuntarios en 2 meses y mejorando progresivamente la marcha hasta ser capaz de caminar sin apoyo a los 7 meses. La dexametasona se redujo paulatinamente hasta su suspensión a los cuatro meses y se mantuvo el clonazepan.

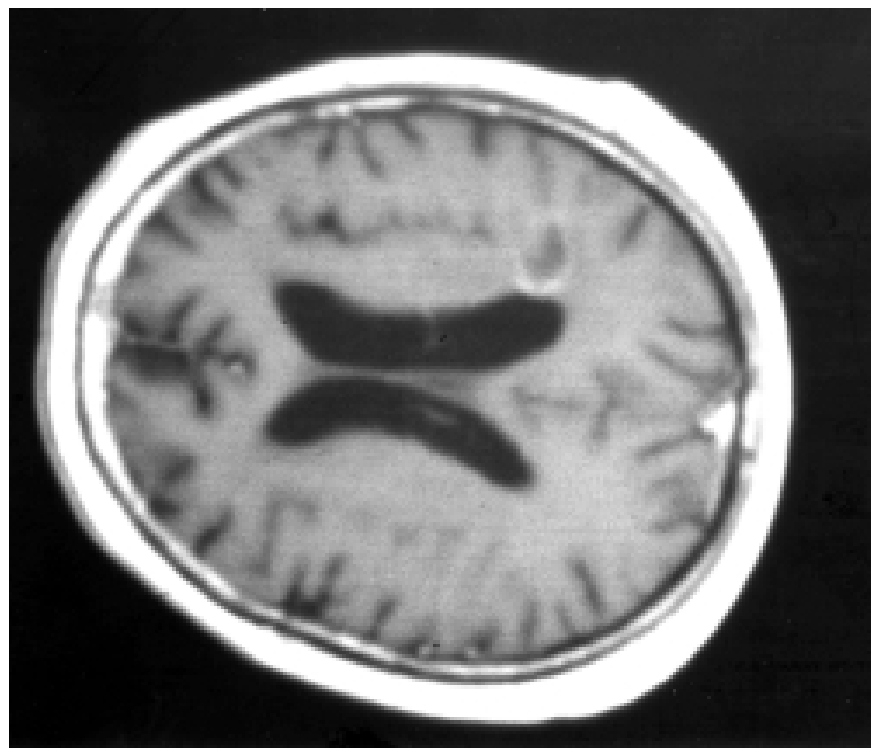

Fig. 1. RM N cerebral. Secuencia axial en T1. M etástasis en sustancia blanca profunda de lóbulo parietal izquierdo que tras la administración de contraste presenta realce anular en su periferia.

\section{DISCUSIÓN}

El opsoclonus puede ser diagnosticado por la presencia de movimientos sacádicos espontáneos, de gran amplitud ocurriendo en todas las direcciones de la mirada sin intervalos sacádicos. La combinación de opsoclonus y movimientos mioclónicos de la cabeza y miembros encontrados en nuestro paciente son típicos del SOM. El SOM se ha descrito en casos aislados asociado a diferentes etiologías: infección vírica o bacteriana del SNC, tumor intracraneal con invasión de tronco encefálico, hemorragia en protuberancia o tálamo, traumatismo craneoencefálico, hidrocefalia, esclerosis múltiple, intoxicaciones (litio, amitriptilina) y como hiperosmolar no cetósico $(1,2)$. El SOM paraneoplasico ha sido descrito en niños como manifestación de neuroblastoma. En adultos es menos frecuente y ha sido observado de forma ocasional asociado a varios tumores: mama, ginecológico, vejiga, melanoma, tiroides, renal, tímico, neurofibrosarcoma, condrosarcoma, enfermedad de Hodgkin y oat cell de pulmón $(3,4)$ (Tabla I).

\section{TABLA I}

\section{CAUSAS DEL SINDRO M E O PSO CLO NUS-M IO CLO NUS}

Infección vírica y bacteriana del SNC

Tumor intracraneal con invasión de tronco encefálico

Hemorragia en protuberancia y tálamo

Isquemia vertebral

Esclerosis múltiple

Hidrocefalia

Coma hiperosmolar

Encefalopatías tóxicas (amitryptilina, haloperidol, litio)

Traumatismo craneoencefálico

Paraneoplásicos: mama, oatt-cell, gástrico, renal, útero, vejiga, tiroides, ovario, páncreas, enfermedad de Hodgkin, melanoma, neuroblastoma (niños) Idiopático

El carcinoma de ovario ha sido relacionado con varios síndromes neurológicos paraneoplásicos: degeneración cerebelosa, polineuropatía sensitivo-motora y polimiositis, pero la asociación con SOM es excepcional $(5,6)$. En nuestro caso, aunque no podemos descartar absolutamente que el cuadro fuese debido a la metástasis parietal, es difícil de asumir esta posibilidad, dada la localización de la lesión respetando tálamo y protuberancia y su reducción considerable con el tratamiento en el momento de iniciarse la sintomatología neurológica. Los citotóxicos y la radioterapia, si bien no pueden descartarse totalmente como responsables del SOM, no hemos encontrado evidencia en la literatura de SOM secundario a estos tratamientos $\mathrm{y}$, además, los citotóxicos fueron administrados tres meses antes del inicio del cuadro. Por tanto, a nuestro juicio la etiología más probable es que el síndrome neurológico fuese una manifestación paraneoplásica del tumor de ovario; teniendo además en cuenta, que al igual que se ha descrito en casos de SOM paraneoplásico recurrente, en nuestro caso el SOM coincide con una reactivación del tumor tras seis años de seguimiento (7). 
A diferencia de otros síndromes paraneoplásicos, anticuerpos antineuronales específicos no son encontrados habitualmente en el SOM paraneoplásico, con la excepción de anticuerpos ant Ri en algunos pacientes con cáncer ginecológico. En nuestra paciente la investigación de anticuerpos anti $\mathrm{Hu}$, anti Ri y anti Yo fueron negativos. A diferencia de los dos únicos casos descritos en la literatura de SOM asociado a cáncer de ovario, en los que el tumor de ovario fue precedido por el síndrome neurológico y la paciente mejoró con el tratamiento de la neoplasia; en nuestro caso, el SOM se inició en el curso evolutivo de la neoplasia ya diagnosticada, coincidió con una reactivación de la enfermedad, se produjo después del tratamiento antitumoral; y, aunque no se puede descartar absolutamente una mejoría espontánea, cronológicamente la paciente mejoró con corticoides y clonazepan, lo que concuerda con algunos casos de SOM paraneoplásico descritos en la literatura (8).

El lugar de la lesión causante del opsoclonus es desconocido. Se ha descrito como resultado de la anormalidad en el control inhibitorio tónico ejercido por las "células pausa" localizadas en la formación reticular pontina paramediana (PPRF) sobre las neuronas que desencadenan los movimientos sacádicos horizontales y verticales. El sitio más probable de lesión es en la región pretectal del tronco. En nuestro caso la RMN cere- bral no reveló anormalidad en esta región (1). La causa del SOM paraneoplasico es desconocida. Sin embargo, la respuesta a la inmunoterapia en algunos casos $(3,8-10)$, la presencia de infiltrados linfocíticos en el SNC en estudios de autopsia (11) y la presencia anticuerpos específicos anti-Ri en pacientes con SOM paraneoplasico y cáncer ginecológico (7) sugiere un mecanismo autoinmune. Se ha sugerido que estos anticuerpos podrían ser directamente responsables de la patogenia del SOM por reacción cruzada entre epitopos tumorales y antígenos neuronales $(12,13)$. Sin embargo, la relación entre anticuerpos antineuronales y cáncer subyacente no es directa ya que estos anticuerpos anti-Ri han sido descritos en pacientes con SOM sin cáncer (8) y a la inversa se han detectado casos de SOM asociado a cáncer sin anticuerpos circulantes (5). La causa de esta disparidad no se conoce e investigaciones futuras determinarán si la diferente respuesta inmunológica del huésped contra el cáncer y la diferente naturaleza histológica del tumor subyacente podrán explicar estas diferencias. A pesar de esta disparidad la presencia de anticuerpos anti Ri o del SOM en un paciente obligaría a investigar la existencia de un cáncer subyacente, fundamentalmente de mama y pulmón. Además, como ocurre en nuestro caso, la presencia de SOM en un paciente diagnosticado de una neoplasia ya tratada obligaría a descartar una reactivación de su enfermedad tumoral.

\section{Bibliografía}

1. Leigh RJ, Zee DS, editors. The neurology of eye movements. 3rd ed. New York: Oxford University Press, 1999.

2. Digre KB. Opsoclonus in adults. Report of three cases and review of the literature. Arch Neurol 1986; 43: 1165-1175.

3. Bataller L, Graus F, Saiz A, Vilchez JJ. Clinical outcome in adult onset idiopathic or paraneoplastic opsoclonus-myoclonus. Brain 2001; 124: 437-443.

4. Posner JB. Paraneoplastic síndromes. In: Neurologic complications of cancer. Davis, Philadelphia 1995: 353-385.

5. Scholz J, Vieregge P, Ruff C. Paraneoplastic opsoclonus-myoclonus syndrome in metastatic ovarian carcinoma (letter). J Neurol Neurosurg Psychiatry 1994; 57: 763-764.

6. Jongen JLM, Moll WJB, Sillevis PAE, Vecht CJ, Tijssen CC. Anti-Ri positive opsoclonus-myoclonus-ataxia in ovarian duct cancer. J Neurol 1998; 245: 691-692.

7. Anderson NE, Budde-Steffen C, Rosenblum MK, et al. Opsoclonus, myoclonus, ataxia and encephalophaty in adults with cancer: A distinct

paraneoplastic syndrome. Medicine 1988; 67: 100-109.

8. Dropcho EJ, Kline LB, Riser J. Antineuronal (anti-Ri) antibodies in a patient with steroid-responsive opsoclonus-myoclonus. Neurology 1993; 43: 207-211.

9. Pless M, Ronthal M. Treatment of opsoclonus-myoclonus with high-dose intravenous immunoglobulin. Neurology 1996; 46: 583584.

10. Das A, Hochberg FH, McNelis S. A review of the therapy of paraneoplastic neurologic syndromes. (Review). J Neurooncol 1999; 41: 181194.

11. Pranzatelli MR. The neurobiology of the opsoclonus-myoclonus síndrome. Clin Neuropharmacol 1992; 15: 186-228.

12. Furneaux HF, Reich LR, Posner JB. Autoantibody synthesis in the central nervous system of patients with paraneoplastic syndromes. Neurology 1990; 40: 1085-1091.

13. Pranzatelli MR. Paraneoplastic syndromes: an unsolved murder. Semin Pediatr Neurol 2000; 7: 118-130. 